\title{
Numerical and Experimental Study of the Squeezing-to-Dripping Transition in a T-Junction
}

\author{
S. Arias - A. Montlaur
}

\begin{abstract}
In this work, we study the transition from squeezing to dripping during the formation of bubbles in a capillary T-junction in conditions relevant to microgravity. The junction is formed by two perpendicular cylinders of equal section ( $1 \mathrm{~mm}$ of internal diameter). The capillary number $\mathrm{Ca}$ (based on the continuous phase) is used as the key parameter of the study. For the range of $C a$ covered in this paper, the same two common bubble formation mechanisms as the ones described in the related literature have been observed: squeezing regime at low $C a$ and dripping regime for higher $C a$. This paper provides a new value of the critical $\mathrm{Ca}$ for the transition from squeezing to dripping. This value has been obtained with two independent approaches, experimentally and numerically. Experimental photographs have been used to determine the value of $C a$ at which a gap appears between the forming bubble and the capillary's wall, as an evidence of the activation of the shearing mechanism related to the dripping regime. Additionally, the dependence of the bubble volume on the capillary number and the gas/liquid flow rate ratio has been analysed. In this work, we also propose a new numerical approach, complementary to the experimental one, carried out with the Computational Fluid Dynamics solver ANSYS Fluent v15.0.7. Numerical simulations have been performed to study the geometry and the behaviour of the gas-liquid interface during the cycle of bubble formation. Upstream the T-junction, as the fluctuation in pressure decreases, the vertical movement of the rear meniscus (gas-liquid interface in contact with the solid vertical capillary) also decreases, and the shear stresses begins to play an active role until overcoming the squeezing mechanism. Numerical simulations presented in this paper support the experimental observa-
\end{abstract}

S. Arias

Escola d'Enginyeria de Telecomunicació i Aeroespacial de Castelldefels, Universitat Politècnica de Catalunya, c/ Esteve Terradas 5, 08860 Castelldefels, Barcelona (Spain)

Tel.: +34 934134129

E-mail: santiago.arias@upc.edu

ORCID: 0000-0001-9233-0178

A. Montlaur

Escola d'Enginyeria de Telecomunicació i Aeroespacial de Castelldefels, Laboratori de Càlcul Numèric (www-lacan.upc.edu), Universitat Politècnica de Catalunya, c/ Esteve Terradas 5, 08860 Castelldefels, Barcelona (Spain)

ORCID: 0000-0002-0243-668X 
tions, confirming that Computational Fluid Dynamics studies can be a useful tool to improve the experimental knowledge.

Keywords Microgravity · Two-phase flow • Bubble generation · T-junction · Computational Fluid Dynamics (CFD) · Squeezing-to-dripping transition

\section{Introduction}

Two-phase flows, both gas-liquid and liquid-liquid, occur constantly in a huge number of scenarios in our daily life. Cosmetic, personal care, food, medical, pharmaceutics or chemical industries are some examples of industries in which two-phase flows have drawn strong attention in the past decades. There is a wide ranging of scientific and technological applications due to their promising great potential and economic revenue, such as chemical reaction, microchemical analysis, mixing process, emulsion technology, medical science, material synthesis, crystalization of proteins and synthesis of nanoparticles.

Microfluidic devices (formed by channels with width and depth typically of the order of $10-100 \mu \mathrm{m}$ ) or slightly larger devices (of the order of $1 \mathrm{~mm}$ ) are usually used to generate monodisperse bubbles and droplets, providing a good controllability of the generation process. In these small devices, gravitational forces generally play a residual role, being overcome by capillary and viscous forces. Since their behaviour is robust to changes in gravity, two-phase flows generated at this scale are also relevant for microgravity environments, making their potential uses expandable beyond on-ground to space-based applications. Some examples can be currently found in life-support systems for human spatial exploration, space bioreactors, thermal management systems and propulsion systems.

An accurate control and management of two-phase flows is then mandatory to guaranty high reliability of these on-ground or space-based applications. A better understanding and a good predictive capacity of the behaviour of two-phase flows are also mandatory. Many devices and methods have been proposed and developed in the past two decades to generate bubbles/droplets. Some reviews can be found in (Utada et al., 2007; Zhao and Middelberg, 2011; Fu and Ma, 2015; Ronshin et al., 2019). All these devices and methodologies share the same major objective: to efficiently control the process of generation of bubbles/droplets. Reaching this goal is necessary in order to be able to regulate the most relevant parameters associated to the process, such as the bubble/droplet size, shape, velocity, spacing between bubbles/droplets and frequency of generation. Of special interest is the technological problem of generating monodisperse colloids, huge quantities of bubbles/droplets (with small size dispersion) immersed in a immiscible continuous phase.

In this work, we focus on the formation of bubbles in a T-junction, a passive crossflow method. This device presents attractive features due to its capability to produce monodisperse bubbles at a high frequency by tuning the flow rate of the continuous and discontinuous phases at the inlets (Thorsen et al., 2001; Arias et al., 2010; Malekzadeh and Roohi, 2015; Arias and González-Cinca, 2016). Many authors have successfully focused on studying the formation of two-phase flows in T-junctions, but there are still some open questions.

Topics such as the evolution of the geometry of the gas-liquid interface during the formation of bubbles, or the physical mechanisms in charge of the generation 
of bubbles remain challenging. Garstecki et al. (2006) described the formation of bubbles/droplets in a microfluidic T-junction with square channels. They identified two regimes, squeezing and dripping, and the corresponding two mechanisms that produce the discontinuous phase breakup with the consequent formation of bubbles/droplets. The squeezing regime is dominated by interfacial forces, whereas the viscous forces exerted by the continuous phase prevail in the dripping regime. The authors provided a simple scaling law for the bubble/droplet size in the squeezing regime:

$$
\frac{L}{w}=1+\alpha \frac{Q_{\text {in }}}{Q_{\text {out }}}
$$

where $L$ is the length of the immiscible slug (drop or bubble), $w$ the width of the channel, $Q_{i n}$ and $Q_{\text {out }}$ are the volumetric flow rates of the dispersed and continuous phases, respectively, and $\alpha$ is a constant of order one, whose particular value depends on the geometry of the T-junction. Eq. 1 evidences that in the squeezing regime, bubble/droplet formation is primarily governed by the flow rate of the dispersed phase relative to the flow rate of the continuous phase, while fluids' properties play little role. As a consequence, the resulting bubble/droplet volume primarily (or the equivalent normalized length $L / w$ ) depends on the ratio of flow rates, $Q_{i n} / Q_{o u t}$, and, to a much lesser extent on the continuous phase's properties.

In his seminal paper, De Menech et al. (2008) studied the transition from squeezing to dripping regime using Computational Fluid Dynamics (CFD). They used the capillary number based on the continuous phase $C a$ as the key parameter of the study $\left(\mathrm{Ca}=\mu_{c} U_{c} / \sigma\right.$, where $\mu_{c}$ and $U_{c}$ are respectively the viscosity and mean velocity of the continuous phase, and $\sigma$ the interfacial tension). When viscous shear starts becoming comparable in magnitude to surface tension, bubble/droplet formation is no longer solely governed by $Q_{i n} / Q_{o u t}$, but also by $C a$, where $Q_{\text {in }} / Q_{\text {out }}$ and $C a$ are two independent control parameters. They suggested that the the squeezing mechanism transitions into the dripping mechanism at a critical value $C a \sim 0.015$, for a viscosity ratio (between the dispersed and continuous phase) of 1 , and at slightly higher $C a$ when the viscosity ratio decreases. According to the authors, at low $C a(C a<0.015)$ the interfacial forces dominate over the viscous forces and the emerging bubble/droplet blocks the main channel. That blockage produces a pressure buildup upstream the slug that pushes the immiscible phase downstream, provoking the phase breakup and the formation of a new bubble/droplet. The squeezing regime is then strongly owed to the effects of the main channel confinement. At moderate $C a(C a>0.015)$, the continuous phase shears off the emerging bubble/droplet, preventing it to entirely fill the main channel. In this second case, the breakup mechanism is mainly due to the shearing forces exerted by the continuous phase that become increasingly important when $C a$ increases. Nevertheless at moderate $C a$, the discontinuous phase still fills a significant part of the main channel, and the effects of channel confinement and pressure buildup upstream the T-junction are still present, although these effects become less important as $\mathrm{Ca}$ increases.

Abate et al. (2012) monitored the pressure fluctuation in a T-junction with Laplace sensors during droplets formation. The authors showed that the pressure of the dispersed phase fluctuates in anti-phase compared to the continuous phase, and that fluctuations persist even at moderate $C a$, confirming the previous observation 
of De Menech et al. (2008). They suggested $C a=0.07$ as the transition value at which the pressure amplitudes begin to diminish rapidly and the viscous forces starts to be dominant.

$\mathrm{Xu}$ et al. (2008) performed experiments and classified the observed two-phase flow patterns by considering the droplets size, correlating these patterns with $C a$. They considered a pure squeezing regime when the bubble length was at least two times larger than the main channel width, and a fully developed dripping regime for lengths smaller than the channel width. The authors suggested that the flow is in transient regime between squeezing and dripping when $0.002<\mathrm{Ca}<0.01$, and therefore the transition would then begin at $C a \sim 0.002$.

van der Graaf et al. (2006) studied droplets detachment both experimentally and with simulations based on the lattice Boltzmann (LB) numerical method. They reported that the influence of the geometry of the main channel decreases when $C a>0.1$, suggesting this value as the beginning of the transition to the dripping regime. With a similar LB approach, Liu and Zhang (2009) performed two dimensional numerical simulations and found the transition at a critical capillary number of 0.018 . They showed in their numerical simulations that the critical value was independent of the flow rate ratio, the viscosity ratio and interestingly of the wetting conditions. They measured the dependence of the droplet diameter on the capillary diameter and found a steeper slope on that dependency around the value $C a=0.018$, suggesting a large effect of the shearing stresses beyond this value.

Guo and Chen (2009) used a volume of fluid (VOF) method to capture the gasliquid interface and compared these numerical results with experiments. The authors studied the velocity distribution inside the dispersed and continuous phases during the bubble formation and correlated these velocities and the interface behaviour with the squeezing and dripping regimes, suggesting a transition number $C a<5.8 \cdot 10^{-3}$. They reported the formation of a circulation of toroidal vortex (inside the continuous phase) while the bubble blocks the main channel (squeezing regime), whereas the continuous phase tends to flow along the interface in the dripping regime. Oishi et al. (2009) presented similar results than Guo and Chen (2009) but using an experimental Particle Image Velocimetry (PIV) technique, allowing them to analyze the dynamical behaviour of both phases separately. They focused on the importance of the gap between the to-be-dispersed phase and the main channel wall, clarifying the dynamical structure of the two-phase flow. They found a change in the velocity distribution around $C a=0.003$, suggesting this value as the transition $\mathrm{Ca}$.

The related literature provides a large variety of values for the critical $C a$, most of them ranging from the order $10^{-3}$ to $10^{-1}$, of which the above examples are only a brief selection. One of the reasons for this diversity may be due to the fact that these studies were carried out in very diverse conditions (experimental vs. numerical simulations, two dimensional vs. three dimensional numerical simulations, gas-liquid vs. liquid-liquid interface, channels with square/rectangular vs. circular sections, dimensions of the channels, etc), and using very different methods to determine the transition itself.

The research question on the squeezing-to-dripping transition is not fully resolved in literature, and an answer is of interest and of relevance for practical applications. The objective of this work is thus to analyze the squeezing-to-dripping transition in a given T-junction bubble generator, formed by cylindrical channels ( $1 \mathrm{~mm}$ of internal diameter). We suggest a new value of the critical $C a$ supported 
by experimental evidences. Afterwards, we complement these experimental observations by analyzing the behaviour of the vertical fluctuation of the rear meniscus (gas-liquid interface in contact with the wall of the vertical capillary) using CFD.

Recently, the authors of the present work introduced 3D numerical studies of the bubble generation process into a T-junction, obtained with the CFD solver ANSYS Fluent v15.0.7. First, comparison of numerical simulations with experimental data showed that numerical data agreed with experimental results from a qualitative point of view, but revealed quantitative discrepancies (Arias and Montlaur, 2017). Then, the influence of the contact angle boundary condition was established by Arias and Montlaur (2018). In this second study, the numerical model was validated and it showed that CFD simulations accurately reproduced experimental results both from qualitative and quantitative points of view, and confirmed that numerical results were very sensitive to the gas-liquid-wall contact angle boundary conditions. Nevertheless, the analysis of the squeezing-to-dripping transition was not part of these previous studies, and is the main contribution of the present work. Section 2 describes the problem statement and methodology for both experimental and numerical approaches. Section 3 presents the main results of this work, whereas conclusions are drawn in Section 4.

\section{Problem statement and methodology}

The experiments consist in generating trains of non-wetting bubbles, by injecting air and distilled water in perpendicular directions into $1 \mathrm{~mm}$ internal diameter capillaries. The liquid capillary is the main channel, with a total length of $10 \mathrm{~mm}$. The gas capillary intersects perpendicularly (vertically) to the main channel. Air and water are considered incompressible and under isothermal conditions. Standard values at $25^{\circ} \mathrm{C}$ are used for density $\left(\rho_{G}=1.225 \mathrm{~kg} / \mathrm{m}^{3}\right.$ and $\rho_{L}=10^{3} \mathrm{~kg} / \mathrm{m}^{3}$, for gas and liquid respectively), viscosity $\left(\mu_{G}=10^{-5}\right.$ Pa.s and $\mu_{L}=10^{-3}$ Pa.s $)$ and gas-liquid surface tension $(\sigma=0.072 \mathrm{~N} / \mathrm{m})$. Gas and liquid superficial velocities (used instead of volumetric flow rates, $Q_{G}$ and $Q_{L}$ ) are defined as $U_{S G}=Q_{G} / A$ and $U_{S L}=Q_{L} / A$, where $A$ is the capillary cross-section area.

Dimensionless numbers characterizing the current problem are summarized in Table 1. Bo is the Bond number, defined as $\rho_{L} g \phi_{c}^{2} / \sigma$, with the gravitational acceleration $g$ and the capillary internal diameter $\phi_{c}$. We is the Weber number, defined as $W e=\rho_{G} \phi_{c} U_{G}^{2} / \sigma$, for a gas (or bubble) velocity $U_{G}$. Re is the Reynolds number, defined as $R e=\rho_{L} \phi_{c} U_{M} / \mu_{L}$, where $U_{M}$ is the mixture superficial velocity, sum of the gas and liquid superficial velocities. Finally, the capillary number $C a$, fundamental parameter to identify the discontinuous phase's breakup, is defined, as usual, as $C a=\mu_{L} U_{S L} / \sigma$.

As shown by the experimental values in table 1, gravity plays a negligible role during the formation of bubbles with respect to capillary effects $(B o<0.29$, (Suo and Griffith, 1964)). Consequently, the present analysis can be assumed as gravity independent. Capillary forces also overcome inertial forces $(W e<2$, (Rezkallah, 1996)), and most of the values of $R e$ correspond to flows under laminar conditions. Therefore, the bubble generation phenomena studied in this work can be considered as mainly controlled by surface tension and the viscous forces exerted by the liquid. The influence of $C a$, which represents the relative effect between the viscous forces and surface tension, will be discussed in depth in Section 3. 
Table 1 Characteristic experimental values

\begin{tabular}{lllllll}
\hline & $\begin{array}{l}U_{S G} \\
{[\mathrm{~m} / \mathrm{s}]}\end{array}$ & $\begin{array}{l}U_{S L} \\
{[\mathrm{~m} / \mathrm{s}]}\end{array}$ & $B o$ & $W e$ & $R e$ & $C a$ \\
\hline $\begin{array}{l}\text { Minimum } \\
\text { value }\end{array}$ & 0.005 & 0.042 & 0.14 & $1.2 \cdot 10^{-4}$ & 70 & $6 \cdot 10^{-4}$ \\
$\begin{array}{l}\text { Maximum } \\
\text { value }\end{array}$ & 1.460 & 0.955 & 0.14 & $1.4 \cdot 10^{-1}$ & 2415 & $1.33 \cdot 10^{-2}$ \\
\hline
\end{tabular}

\subsection{Experiments}

Experiments were performed maintaining $U_{S G}$ constant while increasing $U_{S L}$, leading to an increase of $\mathrm{Ca}$. The range of values of these parameters can be found in table 1 . For each pair $U_{S L}-U_{S G}$, images were taken using a high-speed camera at 4000 frames per second (f.p.s), once the flow had stabilized. More detailed information on the experimental setup can be found in (Arias and González-Cinca, 2016).

The bubble generation frequency is defined as $f=1 / T$, where $T$ is the total time required to form and detach a single bubble. $T$ was computed by counting the number of frames required to form a single bubble, that is, $T=$ \#frames/4000f.p.s. The bubble volume of each single bubble $V_{B}$ was estimated taking into account a simple mass conservation equation,

$$
Q_{G}=f \cdot V_{B},
$$

which leads to $V_{B}=Q_{G} \cdot T$. The bubble (or gas) velocity $U_{G}$ was measured directly over the calibrated images, by considering the displacement of the foremost part of a single bubble.

In T-junctions as the one studied in this work, $f$ follows a characteristic closeto-linear tendency at a very low gas flow rate, then progressively curves until reaching a constant saturation value $f_{\text {sat }}$ when increasing the gas flow rate (Arias et al., 2009). Consequently, two main different regimes arise during the bubble generation process: the linear regime, characterized by its initial slope $a_{0}$ (i.e., slope when $U_{S G}=0 \mathrm{~m} / \mathrm{s}$ ), and the saturation regime, characterized by $f_{\text {sat }}$. Arias and Montlaur (2017) proposed a simple function aiming to smoothly connect the linear part to the constant saturation value, which depends only on the parameters $a_{0}$ and $f_{\text {sat }}$ (see fig. 1 ):

$$
f=f_{\text {sat }}\left(1-e^{\left(-\frac{a_{0}}{f_{s a t}} U_{S G}\right)}\right)
$$

The authors reported that $a_{0}$ is related to the minimum bubble volume that can be generated for each given $U_{S L}$ in the following way: $\left.V_{B}\right|_{\min }=A / a_{0}$. Normalizing $\left.V_{B}\right|_{\text {min }}$ with $A \cdot \phi_{c}$, the minimum volume can be rewritten as $\left.\bar{V}_{B}\right|_{\text {min }}=1 / a_{0} \cdot \phi_{c}$. The other characteristic parameter of eq. $3, f_{\text {sat }}$, is related to the minimum time required to form a bubble, again for each given $U_{S L}$. Therefore, both parameters have a clear physical meaning. Arias et al. (2010) confirmed and reported that both of them increase as $U_{S L}$ does, and consequently the minimum bubble volume decreases with the increase of liquid superficial velocity. Specifically, $f_{\text {sat }}$ was found to follow a linear tendency with $U_{S L}$ : 


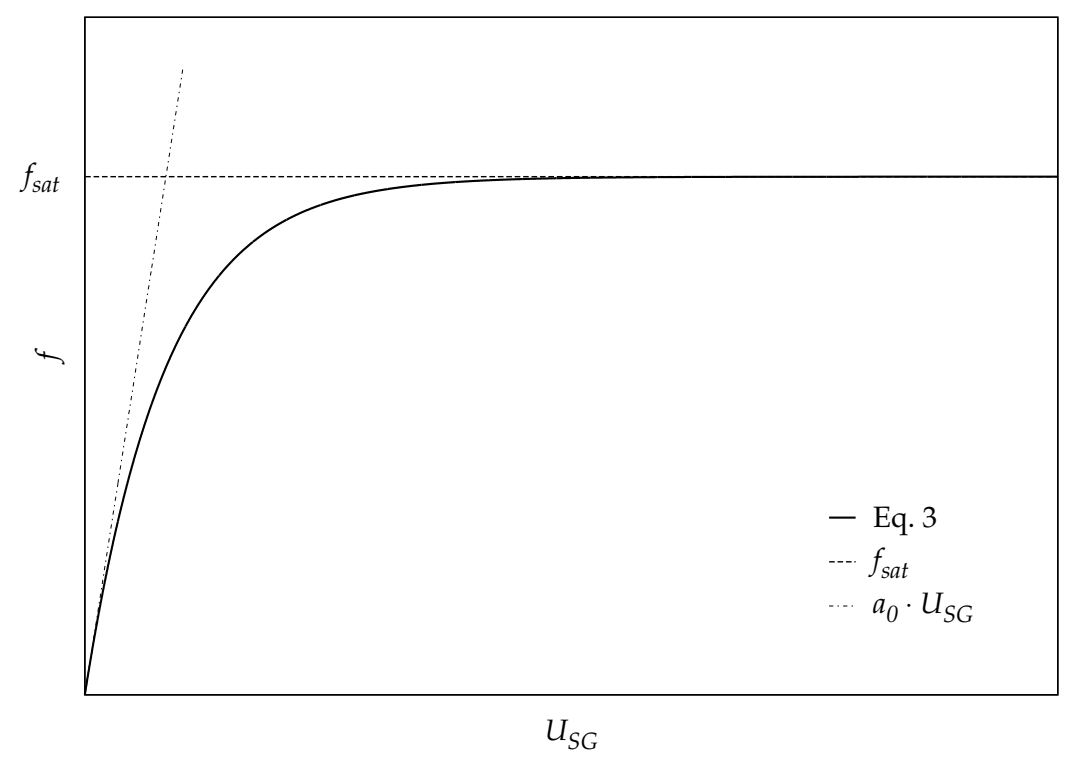

Fig. 1 Bubble generation frequency as a function of the gas superficial velocity, showing the linear and the saturation regimes for a constant $U_{S L}$

$$
f_{\text {sat }}=719.6 \cdot U_{S L}
$$

Eq. 4, combined with the previous mass conservation expressed by eq. 2, led Arias and González-Cinca (2016) to a new normalized expression for the bubble volume, as a function of the ratio of the gas-liquid superficial velocities, in the saturation regime:

$$
\bar{V}_{B}=1.39 \cdot \frac{U_{S G}}{U_{S L}}
$$

where $V_{B}$ was normalized again with $A \cdot \phi_{c}$, and 1.39 is a constant and intrinsic value, which depends on the continuous and discontinuous phases' physical properties, as well as on the T-junction's geometry. More information about this specific value can be found in (Arias and González-Cinca, 2016). Note that eq. 5 is valid only for the saturation regime, and depends on the ratio of gas and liquid flow rates, regardless of the individual value of $U_{S L}$, that is, $\bar{V}_{B}$ does not depend only on the capillary number inside the saturation regime. According to these observations, and always for a given value of $U_{S L}$, the bubble volume is expected to increase starting from $\left.\bar{V}_{B}\right|_{\text {min }}$ in the linear regime, and to continue increasing within the saturation regime following the tendency predicted by eq. 5 . 


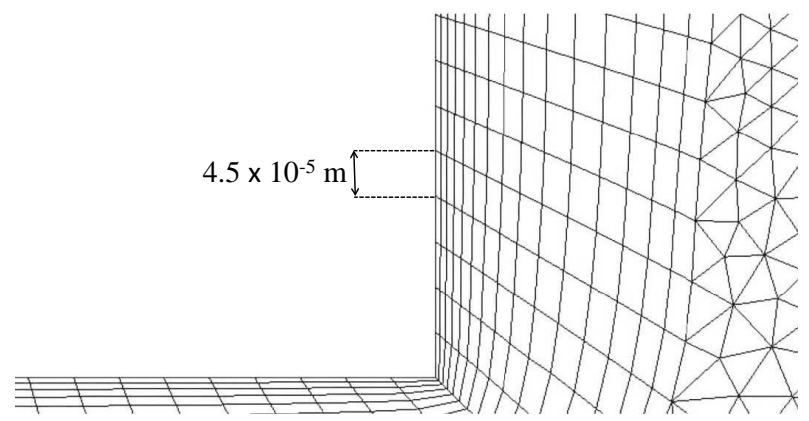

Fig. 2 Close-up view of the hybrid mesh in the vertical wall

\subsection{Numerical simulations}

Numerical simulations presented in this work use the commercial CFD solver ANSYS Fluent v15.0.7. Air and water are modeled as immiscible fluids by using a three-dimensional VOF method and tracking the volume fraction of each fluid, as well as the interface between the phases, throughout the domain. An explicit formulation is used for the VOF model, the PRESTO! (PREssure STaggering Option) scheme for pressure interpolation (ANSYS, 2014), a second-order upwind scheme for spatial discretization and a first-order implicit scheme for the transient formulation. Regarding the boundary conditions, air and water inlets are set as velocity inlets, imposing the corresponding values of $U_{S G}$ and $U_{S L}$ for each case of study, and the outlet of the T-junction as a pressure outlet. All walls are treated as no-slip smooth walls with identical constant contact angle, defining it as the angle between the wall and the tangent to the interface at the wall (measured from the liquid toward the gas phase). Arias and Montlaur (2018) proved it was a non trivial and yet a critical boundary condition for accurate results. All gas and liquid physical properties are set to the same values as the experimental ones. Gravity is set to 0, accordingly to the experimental small value of the Bond number. All details concerning this CFD solver and the settings used for the numerical simulations can be found in (Arias and Montlaur, 2017, 2018).

The computational mesh is a hybrid of 413000 tetrahedral (in the core of the domain) and hexahedral (close to the walls) elements. Note that very fine mesh was used close to the wall: the size of the surface mesh on all walls of the T-junction was set at $4.5 \times 10^{-5} \mathrm{~m}$, and an inflation of 15 layers was created with a first layer of $5.6 \times 10^{-6} \mathrm{~m}$. Figure 2 shows the details of the mesh in the vicinity of the vertical wall of the T-junction. Note that in this area, vertical separation of nodes is thus of $4.5 \times 10^{-5} \mathrm{~m}$, so when measuring the meniscus's vertical position in Section 3.2, every value falling below half this value will be considered within the error margin. Finally $\Delta t=5 \times 10^{-6} \mathrm{~s}$ is selected as the time step for all simulations; see mesh size and time step validations in (Arias and Montlaur, 2017).

Note that, as previously commented, imposing correct boundary conditions on the walls was a key point of the study presented in (Arias and Montlaur, 2018) and allowed to obtain much smaller errors for numerical results with respect to experiments, than the ones first obtained in (Arias and Montlaur, 2017). Indeed, when calculating bubble frequency, velocity and volume, maximum errors were 
all found around 3\%, which is close to the precision margin of the chosen mesh and time step; see (Arias and Montlaur, 2018) for the complete description of the validation of the numerical simulations.

\section{Results and discussion}

In subsection 3.1, we first present the results extracted from experimental data. In subsection 3.2, we then analyze the evolution of the geometry of the discontinuous phase during the formation of bubbles. In this subsection, we use results obtained with numerical simulations to complement the experimental observations.

\subsection{Experimental evidences}

Snapshots shown in fig. 3 were extracted from experiments. In order to allow a correct temporal comparison, all of them correspond to the same instant, just before the gas breakup. Two different values of the gas superficial velocity $(0.106$ and $0.470 \mathrm{~m} / \mathrm{s}$ ) are presented, as well as several values of the capillary number ranging from $6 \cdot 10^{-4}$ to $1.33 \cdot 10^{-2}$. As $C a$ increases (in our experiments by increasing $U_{S L}$ ) bubble sizes decrease as expected (Arias et al., 2010; Arias and González-Cinca, 2016). Likewise, for a given capillary number, bubbles grow as the gas flow rate increases. Also, fig. 3 shows how the gas breakup mechanism changes significantly when the capillary number increases. For small capillary numbers (see for example $C a=0.6-1.5 \cdot 10^{-3}$ ) the interfacial forces overcome the viscous forces. As a result, the emerging gas entirely fills the main channel. The forming bubble blocks the main channel, preventing the liquid from passing through the Tjunction. The buildup of pressure upstream the bubble, caused by the confinement of geometry in the main channel, pushes the gas forward provoking the gas breakup and the detachment of a bubble.

For higher $C a$, when the shear stress exerted by the liquid exceeds a critical value, the squeezing-to-dripping transition takes place. Under these circumstances, the incoming gas fails to block entirely the main channel (De Menech et al., 2008; Guo and Chen, 2009; Liu and Zhang, 2009; Yeom and Lee, 2011), even for larger values of gas flow rate (see for example $C a=7.4-13.3 \cdot 10^{-3}$ ). The liquid flows through the gap existing between the forming bubble and the bottom wall, shearing off the emerging bubble and preventing the gas to fully fill the main channel. Thus, the appearance of this gap indicates the beginning of the transition, for which the shearing effect starts playing a major role, releasing the pressure buildup upstream. Nevertheless, the squeezing mechanism persists, although decreasing in intensity as the viscous forces become dominant. Therefore, both mechanisms coexist for a range of values of the capillary number (De Menech et al., 2008; Liu and Zhang, 2009; Abate et al., 2012).

Determining the exact critical value of $C a$ at which the shearing mechanism begins to be dominant remains a challenging task. Based upon experimental observations of the appearance of the gap between the bubble and the bottom wall (see fig. 3), the squeezing-to-dripping transition must start at a value of $C a$ in the range $1.5-5.9 \cdot 10^{-3}$ for the specific T-junction and fluids studied here. Looking carefully at fig. 3 , this transition seems to be around the value $C a=4.4 \cdot 10^{-3}$. 


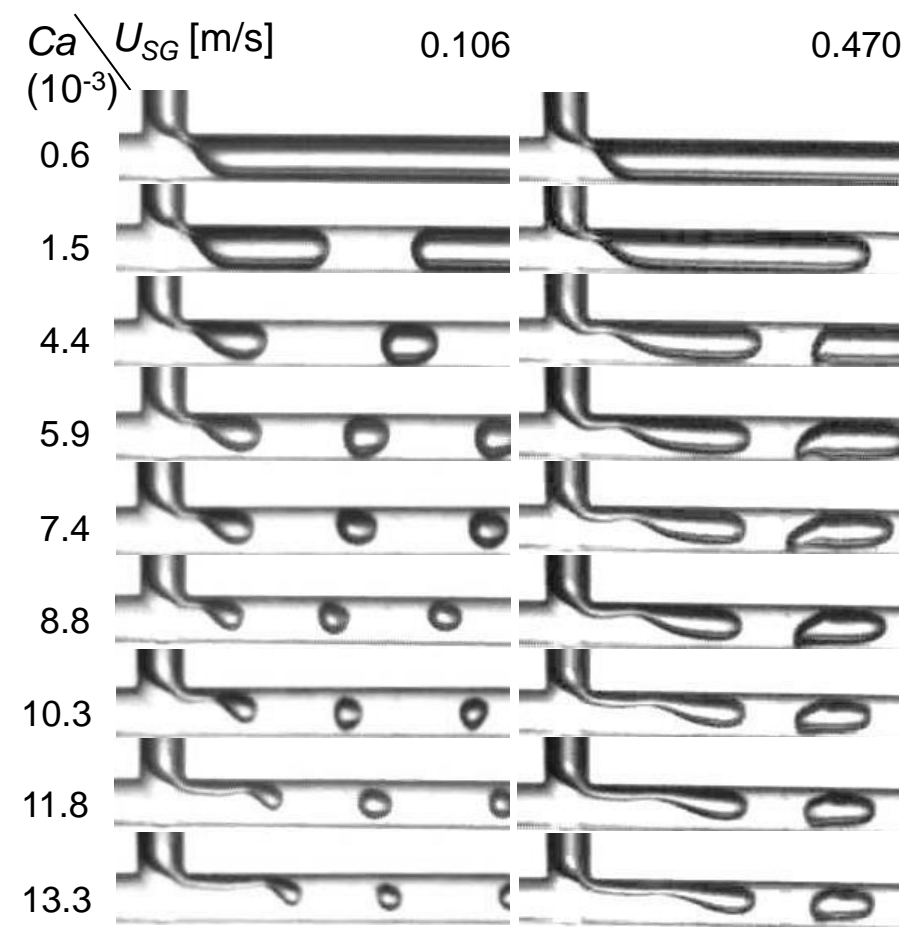

Fig. 3 Gas breakup for two gas superficial velocities at several capillary numbers

Fig. 4 shows the normalized bubble volume as a function of the ratio of the gas/liquid volumetric flow rates (i.e. superficial velocities), for several values of $C a$. The figure includes the asymptotic tendency followed by $\bar{V}_{B}$ into the saturation regime (solid line), as described by eq. 5 . For each value of $U_{S G} / U_{S L}, \bar{V}_{B}$ tends to a constant value $\left(1.39 \cdot U_{S G} / U_{S L}\right)$ as $C a$ increases. At low $U_{S G} / U_{S L}, \bar{V}_{B}$ leans toward a minimum value $\left.\bar{V}_{B}\right|_{\text {min }}$, which depends on $C a$. As $U_{S G} / U_{S L}$ increases, $\bar{V}_{B} \mid$ tends to eq. 5 . These two tendencies are identified as the linear regime (leading to $\left.\bar{V}_{B}\right|_{\min }$ ) and the saturation regime (leading to the asymptotic tendency defined by eq. 5 ), as explained in section 2.1 .

Combining eqs. 2-3, and taking also into account the identity $\left.\bar{V}_{B}\right|_{\min }=1 / a_{0}$. $\phi_{c}$, a new expression of $\bar{V}_{B}$ as a function of $U_{S G} / U_{S L}$ can be obtained:

$$
\bar{V}_{B}=\frac{1.39 \cdot \frac{U_{S G}}{U_{S L}}}{1-e^{\left(-\frac{1.39}{\left.\bar{V}_{B}\right|_{\min }} \frac{U_{S G}}{U_{S L}}\right)}}
$$

Note that $\bar{V}_{B}$ depends explicitly on $U_{S G} / U_{S L}$ and implicitly on $C a$ (through $\left.\left.\bar{V}_{B}\right|_{\text {min }}\right)$. Therefore, eq. 6 tends to $\left.\bar{V}_{B}\right|_{\min }$ for low $U_{S G} / U_{S L}$, and to $1.39 \cdot U_{S G} / U_{S L}$ for high $U_{S G} / U_{S L}$, in the same manner as eq. 1 tends to a constant value (1) and $\alpha \cdot U_{S G} / U_{S L}$, respectively. Both equations behave similarly, but the points located in the intermediate region between these two tendencies fit eq. 6 better.

Nevertheless, the scaling law of Garstecki et al. (2006), recalled in eq. 1, does not capture the capillary number dependency of $L / w$, since accordingly to eq. 1 , $L / w$ should always tend to 1 . However, the normalized bubble volume shown in 


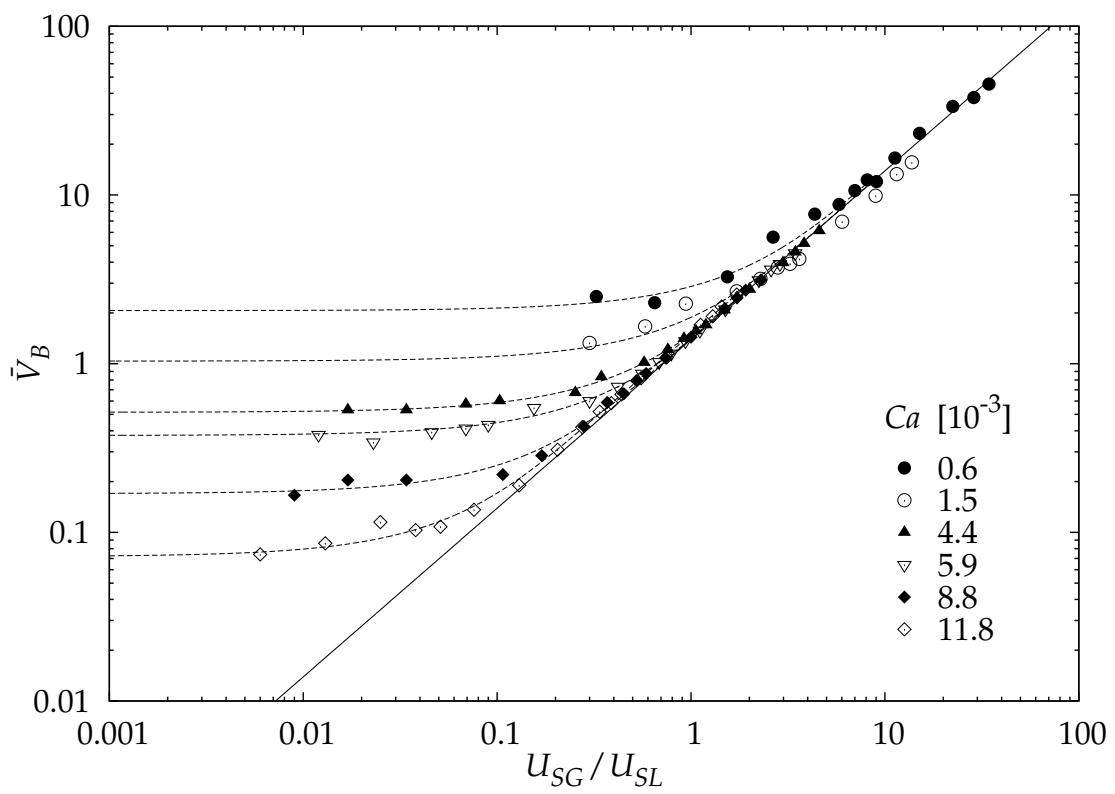

Fig. 4 Normalized bubble volume as a function of $U_{S G} / U_{S L}$ for several values of $C a$. Symbols correspond to experimental data, solid line to eq. 5, and dashed lines to eq. 6 (particularized for each $\mathrm{Ca}$ shown in the figure)

fig. 4 shows that instead of always tending to 1 , as the capillary number increases, the minimum volume continues decreasing until a value that does not depend on $U_{S G} / U_{S L}$. This variation of $\left.\bar{V}_{B}\right|_{\min }$ with $C a$ was already observed by some authors (van der Graaf et al., 2006; Christopher et al., 2008; Xu et al., 2008).

Since the bubble frequency behaves similarly for all $C a$ studied in this work (linear regime followed by a saturation regime), eq. 6 is expected to indistinctly describe the behaviour of $\bar{V}_{B}$ in the squeezing and the dripping regimes. See for example how lines corresponding to capillary numbers of $0.6 \cdot 10^{-3}$ and $11.8 \cdot 10^{-3}$ behave similarly (the first case corresponds to the squeezing regime, while the second one is a clear example in which the shearing forces have been activated; see again fig. 3). Therefore, fig. 4 does not seem to be the most suitable figure to capture the value of $C a$ at which the squeezing-to-dripping transition begins.

Fig. 5 shows $\left.\bar{V}_{B}\right|_{\text {min }}$ for each $C a$ of fig. 4 as a function of $C a$. The points of fig. 5 correspond then to similar and very low values of the ratio $U_{S G} / U_{S L}$. In fact, below $U_{S G} / U_{S L}=0.01,\left.\bar{V}_{B}\right|_{\text {min }}$ does not change appreciably for the $C a$ values of fig. 4 . The tendencies observed in fig. 5 are comparable to those already reported in the related literature (De Menech et al., 2008; Liu and Zhang, 2009). At low $C a$, the bubble minimum volume is not very much affected by the capillary number, that is, it mainly depends on the ratio of the gas/liquid flow rates. But for moderate $C a$, and beyond a $C a$ critical value, the minimum volume experiences steeper variation, being mainly affected by the increase of the capillary number. Fig. 5 includes two lines as a guide to the eye, to help capturing both tendencies. Though the exact intersection point of both lines is prone to experimental error, 


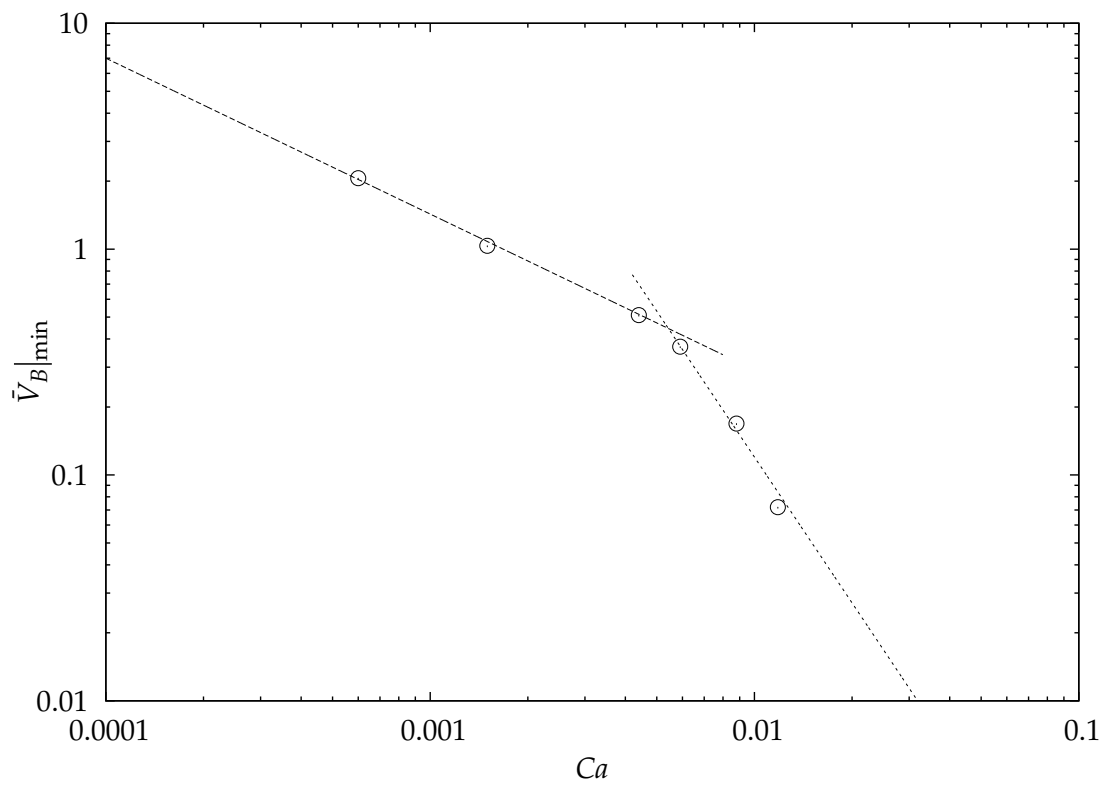

Fig. 5 Normalized bubble volume as a function of $C a$ for very low $U_{S G} / U_{S L}$. Dashed lines are used to guide the eye

the change in tendency occurs again in the vicinity of the value $4.4 \cdot 10^{-3}$. This result is thus in good agreement with previous observations from fig.3, showing the appearance of the gap between the bubble and the bottom wall around this capillary number.

\subsection{Study of the geometry of the gas-liquid interface}

For low $\mathrm{Ca}$, the gas-liquid interface is in quasi-equilibrium along the bubble generation process. The deformation of the gas-liquid interface (due to the viscous shear stress) can be considered as negligible (Garstecki et al., 2006; van Steijn et al., 2010). Then, while the shape of the bubble's main body is caused by the gas confinement in the main channel, the back interface tends to be as much spherical as possible in order to minimize the total surface energy. This rear shape is perceived in frontal two-dimensional pictures as a circle segment, tangential to both the vertical and horizontal capillaries (see fig. 6). As the upstream pressure increases, the liquid pushes the interface downstream towards the wall corner, while the back interface tries to maintain its circular shape and the radius of this curvature increases (van Steijn et al., 2009), until it finally breaks at sharp corner (De Menech et al., 2008; Guo and Chen, 2009; Liu and Zhang, 2009), or close to it. We have observed in our experiments that even for small values of $C a$, the breakup point can displace downstream as the gas superficial velocity increases (see $C a=0.6-1.5 \cdot 10^{-3}$ for $U_{S G}=0.47 \mathrm{~m} / \mathrm{s}$ in fig. 3 ). 


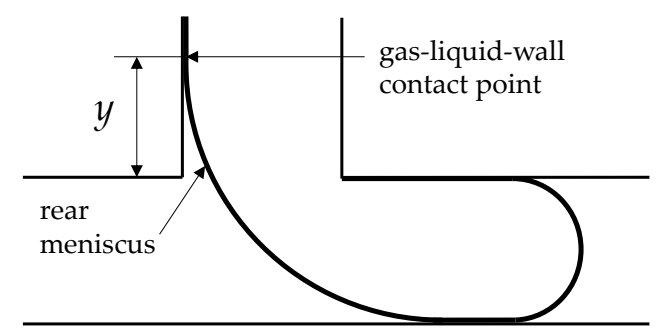

Fig. 6 Sketch of the T-junction, showing the formation of one bubble, the rear meniscus and the $y$ position of the gas-liquid-wall contact point

At the same time that the liquid pushes the back interface forward, the rear meniscus rises, reaching a maximum elevation just in the breakup moment, in which the back interface is more stretched. In other words, the rise of the rear meniscus indicates that the liquid enters the gas channel (vertical capillary), pushing the meniscus upward. Just after the bubble detachment, the pressure upstream drops, causing the liquid to move along the main channel. The gas moves again towards the main channel pulling down the gas-liquid interface until the new forming bubble blocks once more the main channel and a new formation cycle begins (Arias et al., 2012).

The behaviour and geometry of the gas-liquid interface described above changes noticeably for higher $\mathrm{Ca}$. As the capillary number increases, the liquid's viscous shear stresses force the breakup point to move downstream (see for example $\mathrm{Ca}=$ $8.8-10.3 \cdot 10^{-3}$ in fig. 3 ). When this point displaces even more downstream, the dripping-to-jetting transition takes place (De Menech et al., 2008; Li et al., 2012; Nunes et al., 2013). The back interface is also affected, loosing its circular shape. The liquid passes through the gap between bubble and wall during the entire formation cycle, the pressure fluctuation upstream decreases and the rear meniscus stabilizes. Hence, if the vertical fluctuation of the rear meniscus indicates the predominance of the squeezing mechanism, its stabilization must on the contrary correspond to an increase in importance of the shearing mechanism (Oishi et al., 2009). That is, the vertical fluctuation of the rear meniscus gives a visual measure of the pressure buildup upstream. In this work, we choose to focus on the analysis of this vertical fluctuation instead of the circularity of the rear meniscus.

Figs. 7 and 8 show the formation cycle of a bubble at different temporal stages. Having validated the numerical code against experimental results (see subsection 2.2 ), it is now possible to compare numerical and experimental results. The dimensionless time $\bar{t}=t / T$ is denoted in each picture, where $t$ is the time from the previous bubble's detachment and $T$ is the time required to form a bubble, as explained in Section 2.1. For the cases presented in figs. 7 and 8, the reference time $T$ is equal to $2.1 \cdot 10^{-2} \mathrm{~s}\left(U_{S G}=0.100 \mathrm{~m} / \mathrm{s}, C a=1.5 \cdot 10^{-3}\right)$ and $8.4 \cdot 10^{-3} \mathrm{~s}$ $\left(U_{S G}=0.081 \mathrm{~m} / \mathrm{s}, C a=4.4 \cdot 10^{-3}\right)$. More details on these two cases can be found in (Arias and Montlaur, 2018). Fig. 7 corresponds to $C a=1.5 \cdot 10^{-3}$ and shows the characteristic behaviour previously described for low $C a$ : the breakup point occurs close to the corner downstream the T-junction and the rear meniscus rises vertically just before the gas breakup. Note how the bubble's rear interface maintains a circular curvature along the first steps of the process (see from $\bar{t}=0$ 


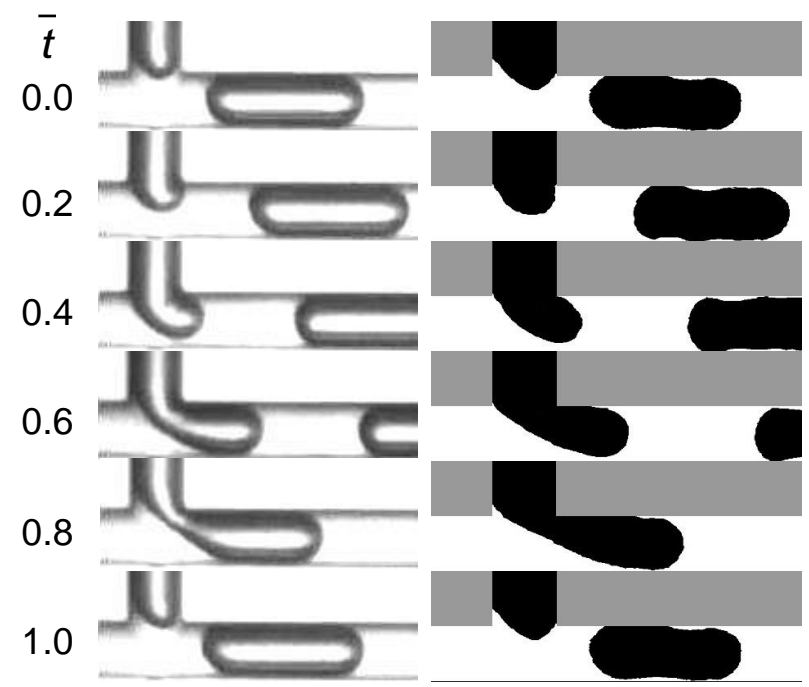

Fig. 7 Formation of a bubble as a function of $\bar{t} . U_{S G}=0.100 \mathrm{~m} / \mathrm{s}, C a=1.5 \cdot 10^{-3}$

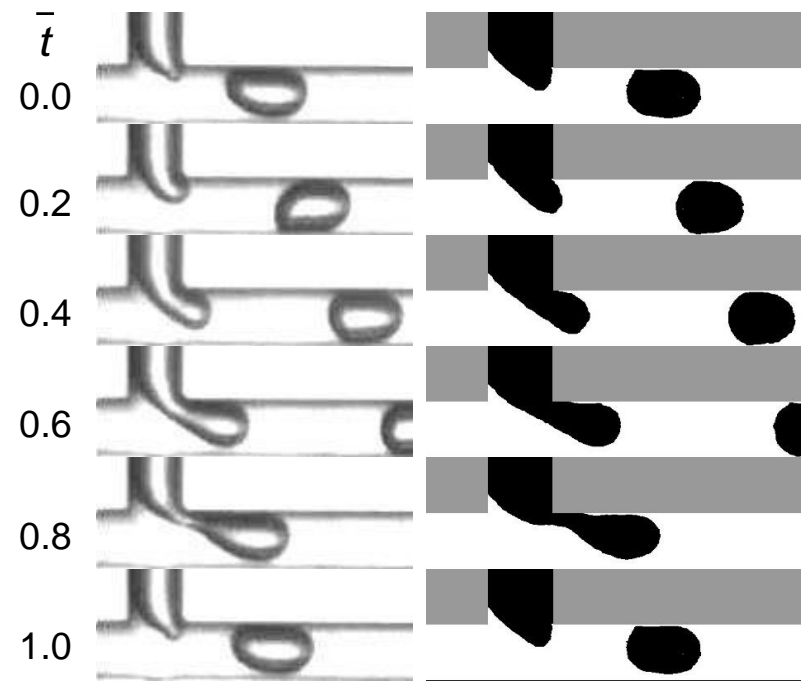

Fig. 8 Formation of a bubble as a function of $\bar{t}$. $U_{S G}=0.081 \mathrm{~m} / \mathrm{s}, C a=4.4 \cdot 10^{-3}$

up to 0.6 ). Fig. 8 corresponds to $C a=4.4 \cdot 10^{-3}$, and in opposition to the anterior case, the breakup point has moved downstream and the rear meniscus seems to be vertically stabilized. In contract to the previous case, the rear meniscus is no longer circular but more stretched instead.

Based on the former comments, we propose a new approach to determine the critical $C a$ value for squeezing-to-dripping transition. We focus on the vertical displacement of the rear meniscus, by analysing the position of the highest point $y$ of the gas-liquid-wall contact point along the bubble generation process. The height $y$ is measured from the corner of the T-junction (see sketch in fig. 6) and normalized 


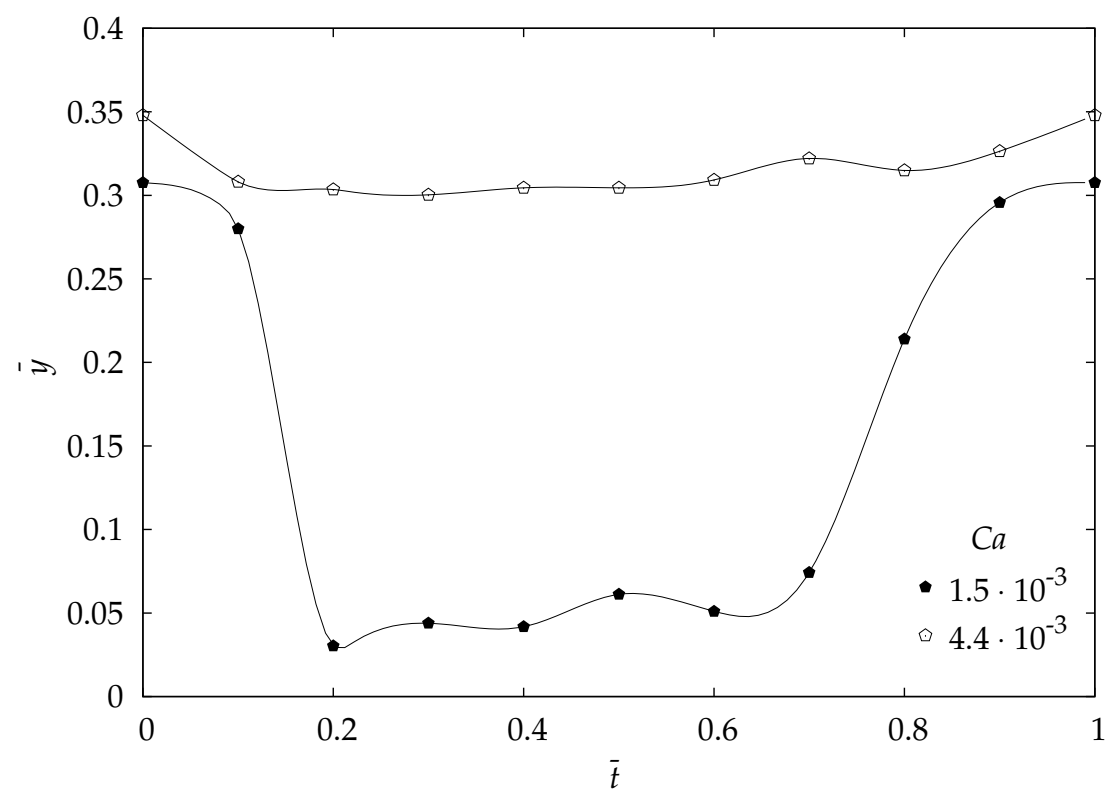

Fig. 9 Maximum value of the vertical position of the rear meniscus $\bar{y}$ (normalized with $\mid p h i$ ) as a function of the dimensionless time $\bar{t}$ for $C a=1.5 \cdot 10^{-3}$ (with $U_{S G}=0.100 \mathrm{~m} / \mathrm{s}$ ) and $C a=4.4 \cdot 10^{-3}$ (with $U_{S G}=0.081 \mathrm{~m} / \mathrm{s}$ ). Lines are guide to the eye

with the capillary diameter. Measuring accurately the position of this point from our experimental pictures is challenging, because the curvature of the vertical cylinder generates shadows in the image, with darker areas just in the meniscus. Since numerical simulations do not present this limitation, we decided to contrast the experimental conclusions extracted in subsection 3.1 with the information provided by CFD analysis. Fig. 9 shows the vertical position of the meniscus' highest point $\bar{y}$ as a function of the dimensionless time $\bar{t}$. The two lines included in fig. 9 correspond to the same cases shown in figs. 7-8, that is, same $C a$ and $U_{S G}$.

For $C a=1.5 \cdot 10^{-3}$, the meniscus reaches its maximum position just during the gas breakup. Afterwards, the gas flows towards the main channel and the meniscus reaches its minimum position. During approximately the interval $\bar{t}=0.2-0.7$, the growing bubble blocks the channel, the liquid pushes the interface forward, and the meniscus moves upward (this evolution can also be seen in fig. 7). When the meniscus reaches again its maximum position, the elongated bubble breaks, and the bubble forming cycle starts again. The behaviour observed for $C a=4.4 \cdot 10^{-3}$ differs from the precedent case. In this situation, the vertical displacement of the meniscus can reasonably be considered negligible.

Fig. 10 presents the interval of the meniscus' normalized vertical position. It has been calculated as the difference between its normalized maximum and minimum positions, i.e., $\bar{y}_{\max }-\bar{y}_{\min }$. The dotted line marks the position of value 0.0225 , which corresponds to half the node separation of the mesh (normalized with $\phi_{c}$ ) in the vertical capillary, as explained in section 2.2. Several gas superficial values have been plotted. Whereas the points corresponding to $C a=1.5 \cdot 10^{-3}$ are above 


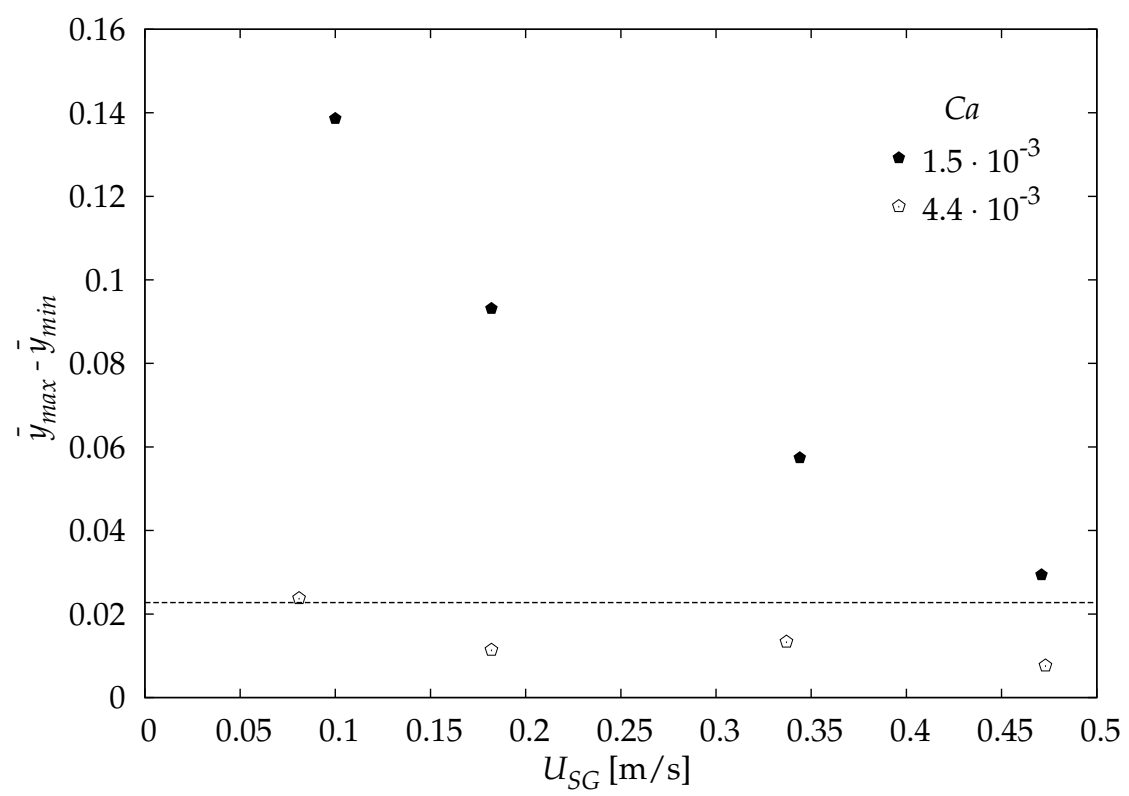

Fig. 10 Interval of the meniscus's vertical normalized positions as a function of $U_{S G}$ for $C a=1.5 \cdot 10^{-3}$ and $C a=4.4 \cdot 10^{-3}$. Dashed line corresponds to half the value of the separation between vertical nodes of the mesh

the value 0.0225 , the ones of $C a=4.4 \cdot 10^{-3}$ are close or below that value, and then within the error margin associated to the mesh size. According to this result, we conclude that for $C a=4.4 \cdot 10^{-3}$, the meniscus is static enough to consider that the shearing mechanism has been activated and the squeezing-to-dripping transition has begun.

$C a=4.4 \cdot 10^{-3}$ differs from other values that can be found in the literature for the squeezing-to-dripping transition. In some cases the quantitative difference is small (Xu et al., 2008; Guo and Chen, 2009; Oishi et al., 2009), whereas in others it can be of one or even two orders of magnitude (De Menech et al., 2008; Abate et al., 2012; van der Graaf et al., 2006; Liu and Zhang, 2009). We consider that the differences can be a consequence of mainly the different methods, fluids, devices and geometries under which these studies were carried out. The biggest difference is probably due to the fact that the studies described in section 1 typically used T-junctions with rectangular/square sections, instead of the circular one studied in this work. In any case, the value presented here, double checked between experiments and numerical simulations, is a new critical value found in the experimental conditions related in this paper.

\section{Conclusions}

This paper studies the squeezing-to-dripping transition, focusing on the generation of bubbles in a T-junction formed by cylindrical capillaries of $1 \mathrm{~mm}$ internal 
diameter. Air and water were used to generate two-phase flows. The small Bond number of the experiments makes the results shown in this work applicable to the field of microgravity.

For the range of $\mathrm{Ca}$ covered in this work, the same two regimes as the ones described in the related literature were observed during the formation of bubbles: squeezing regime at low $C a$ and dripping regime for higher $C a$. This work provides a new value of the critical capillary number, $C a=4.4 \cdot 10^{-3}$, at which the shearing mechanism starts to play a significant role, and therefore the squeezing-to-dripping transition begins. This critical value was confirmed both experimentally and numerically.

Photographic evidences (fig. 3) showed that around the value $C a=4.4 \cdot 10^{-3}$, the gas starts failing to entirely fill the main channel, and a gap is formed between the forming bubble and the bottom wall. The liquid flows through this gap, shearing off the emerging bubble. This evidence was supported by the steeper slope observed after this value of $\mathrm{Ca}$ in the evolution of the normalized bubble minimum volume as a function of the capillary number, for very low values of $U_{S G} / U_{S L}$.

Numerical simulations were carried out to study the gas-liquid interface and its changes in geometry as $\mathrm{Ca}$ increases. The activation of the shearing mechanism showed to have a significant impact on the forming bubble's geometry, causing the gas breakup point to move downstream and the rear meniscus to stop moving vertically. When the meniscus' highest point stays still, the fluctuation in pressure upstream is small enough to consider that the shearing mechanism is playing an active role. To the best of our knowledge, this is the first time that this approach is used to determine the critical $C a$, showing that numerical simulations are a useful tool to improve the experimental knowledge.

The value $4.4 \cdot 10^{-3}$ presented in this paper differs from other squeezing-todripping critical $\mathrm{Ca}$ provided in the literature. In some cases the quantitative difference is small, whereas in others it can be up to even two orders of magnitude. We consider that this disagreement can be mainly due, in addition to the different methods used and the researchers' own subjectivity, to the type of T-junction widely used in the literature, with rectangular (or square) section, instead of the circular one of this work. The value $C a=4.4 \cdot 10^{-3}$ remains then associated to the particular type of $\mathrm{T}$-junction studied in this work.

Acknowledgements This work has been partially supported by the Spanish Ministerio de Economía y Competitividad, Secretaría de Estado de Investigación, Desarrollo e Innovación (Project number MTM2013-46313-R) and the Generalitat de Catalunya (Grant number 2017SGR-1278).

\section{References}

A.R. Abate, M. Pascaline, V. van Steijn, and D.A. Weitza. Experimental validation of plugging during drop formation in a T-junction. Lab on a Chip, 12:1516-1521, 2012. doi: 10.1039/C2LC21263C.

ANSYS. ANSYS Academic Research, Release 15.0,7, Help System. Technical report, ANSYS, Inc., 2014.

S. Arias and R. González-Cinca. Analysis of the characteristic lengths in the bubble and slug flow regimes generated in a capillary $\mathrm{T}$ - 
junction. International Journal of Multiphase Flow, 87:167-174, 2016. doi: 10.1016/j.ijmultiphaseflow.2016.09.011.

S. Arias and A. Montlaur. Numerical study and experimental comparison of twophase flow generation in a T-junction. AIAA Journal, 55(5):1565-1574, 2017. doi: 10.2514/1.J055387.

S. Arias and A. Montlaur. Influence of contact angle boundary condition on CFD simulation of T-junction. Microgravity Sci. Technol., 30(4):435-443, 2018. doi: 10.1007/s12217-018-9605-x.

S. Arias, X. Ruiz, L. Ramírez-Piscina, J. Casademunt, and R. González-Cinca. Experimental study of a microchannel bubble injector for microgravity applications. Microgravity Sci. Technol., 21:107-118, 2009. doi: 10.1007/s12217-0089060-1.

S. Arias, R. González-Cinca, X. Ruiz, L. Ramírez-Piscina, and J. Casademunt. Characterization of the performance of a minibubble generator in conditions relevant to microgravity. Colloids Surfaces A: Physicochem Eng Aspects, 365: 52-57, 2010. doi: 10.1016/j.colsurfa.2010.03.004.

S. Arias, D. Legendre, and R. González-Cinca. Numerical simulation of bubble generation in a T-junction. Computers and Fluids, 56:49-60, 2012. doi: 10.1016/j.compfluid.2011.11.013.

G.F. Christopher, N.N. Noharuddin, J.A. Taylor, and S.L. Anna. Experimental observations of the squeezing-to-dripping transition in T-shaped microfluidic junctions. Physical review E, 78(3):036317, 2008. doi: 10.1103/PhysRevE.78.036317.

M. De Menech, P. Garstecki, F. Jousse, and H.A. Stone. Transition from squeezing to dripping in a microfluidic T-shaped junction. Journal of Fluid Mechanics, 595:141-161, 2008. doi: 10.1017/S002211200700910X.

T. Fu and Y. Ma. Bubble formation and breakup dynamics in microfluidic devices: A review. Chemical Engineering Science, 135:343-372, 2015. doi: 10.1016/j.ces.2015.02.016.

P. Garstecki, M.J. Fuerstman, H.A. Stone, and G.M. Whitesides. Formation of droplets and bubbles in a microfluidic T-junction-scaling and mechanism of break-up. Lab Chip, 6:437-446, 2006. doi: 10.1039/b510841a.

F. Guo and B. Chen. Numerical study on Taylor bubble formation in a microchannel T-junction using VOF method. Microgravity Sci. Technol, 21:51-58, 2009. doi: 10.1007/s12217-009-9146-4.

X.-B. Li, F.-C. Li, J.-C. Yang, H. Kinoshita, M. Oishi, and M. Oshima. Study on the mechanism of droplet formation in T-junction microchannel. Chemical Engineering Science, 69:340-351, 2012. doi: 10.1016/j.ces.2011.10.048.

H. Liu and Y. Zhang. Droplet formation in a T-shaped microfluidic junction. Journal of Applied Physics, 106:034906, 2009. doi: 10.1063/1.3187831.

$\mathrm{S}$. Malekzadeh and E. Roohi. Investigation of different droplet formation regimes in a T-junction microchannel using the VOF technique in OpenFOAM. Microgravity Sci. Technol., 27:231-243, 2015. doi: 10.1007/s12217-015-9440-2.

J.K. Nunes, S.S.H. Tsai, J. Wan, and H.A. Stone. Dripping and jetting in microfluidic multiphase flows applied to particle and fibre synthesis. J. Phys. D: Appl. Phys., 46:114002, 2013. doi: 10.1088/0022-3727/46/11/114002.

M. Oishi, H. Kinoshita, T. Fujii, and M. Oshima. Confocal micro-PIV measurement of droplet formation in a T-shaped micro-junction. Journal of Physics: Conference Series, 147(1):012061, 2009. doi: 10.1088/1742-6596/147/1/012061. 
K.S. Rezkallah. Weber number based flow-pattern maps for liquid-gas flows at microgravity. International Journal of Multiphase Flow, 22(6):1265-1270, 1996. doi: 10.1016/0301-9322(96)00047-X.

F.V. Ronshin, Yu.A. Dementyev, E.A. Chinnov, V.V. Cheverda, and O.A. Kabov. Experimental investigation of adiabatic and pressure drop in slit microchannel. Microgravity Sci. Technol., 31:693-707, 2019. doi: 10.1007/s12217-019-09747-1.

M. Suo and P. Griffith. Two-phase flow in capillary tubes. Journal of Basic Engineering, 86:576-582, 1964. doi: 10.1115/1.3653176.

T. Thorsen, R.W. Roberts, F.H. Arnold, and S.R. Quake. Dynamic pattern formation in a vesicle-generating microfluidic device. Physical Review Letters, 86 (18):4163-4167, 2001. doi: 10.1103/PhysRevLett.86.4163.

A.S. Utada, L.-Y. Chu, A. Fernandez-Nieves, D.R. Link, C. Holtze, and D.A. Weitz. Dripping, jetting, drops, and wetting: the magic of microfluidics. MRS Bulletin, 32(9):702-708, 2007. doi: 10.1557/mrs2007.145.

S. van der Graaf, T. Nisisako, C.G.P.H. Schroën, R.G.M. van der Sman, and R.M. Boom. Lattice Boltzmann simulations of droplet formation in a T-shaped microchannel. Langmuir, 22(9):4144-4152, 2006. doi: 10.1021/la052682f.

V. van Steijn, R. Chris, C.R. Kleijn, and M.T. Kreutzer. Flows around confined bubbles and their importance in triggering pinch-off. Physical Review Letters, 103:214501-4, 2009. doi: 10.1103/PhysRevLett.103.214501.

V. van Steijn, R. Chris, C.R. Kleijn, and M.T. Kreutzer. Predictive model for the size of bubbles and droplets created in microfluidic T-junctions. Lab on a Chip, 10:2513-2518, 2010. doi: 10.1039/c002625e.

J.H. Xu, S.W. Li, J. Tan, and G.S. Luo. Correlations of droplet formation in Tjunction microfluidic devices: from squeezing to dripping. Microfluid Nanofluid, 5(6):711-717, 2008. doi: 10.1007/s10404-008-0306-4.

S. Yeom and S.Y. Lee. Size prediction of drops formed by dripping at a micro T-junction in liquid-liquid mixing. Experimental Thermal and Fluid Science, 35:387-394, 2011. doi: 10.1016/j.expthermflusci.2010.10.009.

C.-X. Zhao and A.P.J. Middelberg. Two-phase microfluidic flows. Chemical Engineering Science, 66(7):1394-1411, 2011. doi: 10.1016/j.ces.2010.08.038. 